\title{
Nonlinear dynamics of spatio-temporal waves in multimode fibres
}

\author{
Stefan Wabnitz ${ }^{1,2}$, Katarzyna Krupa ${ }^{3}$, Alessandro Tonello ${ }^{4}$, Alain Barthelemy ${ }^{4}$, Guy Millot ${ }^{3}$, Daniele \\ Modotto $^{1}$, and Vincent Couderc ${ }^{4}$ \\ 1. Dipartimento di Ingegneria dell'Informazione, Università di Brescia and INO-CNR, Via Branze 38, 25123 Brescia, Italy \\ 2. Novosibirsk State University, 1 Pirogova str., Novosibirsk 630090, Russia \\ 3. Université Bourgogne Franche-Comté, ICB UMR CNRS 6303, 9 Avenue A. Savary, 21078 Dijon, France \\ 4. Université de Limoges, XLIM, UMR CNRS 7252, 123 Avenue A. Thomas, 87060 Limoges, France \\ stefan.wabnitz@unibs.it
}

\begin{abstract}
Nonlinear multimode fibers provide an intriguing test-bed for exploring complex spatio-temporal beam dynamics. We overview recent experimental observations of Kerr beam self-cleaning, parametric sideband series and supercontinuum generation in passive and active multimode optical fibers.

OCIS codes: (190.4370) Nonlinear optics, fibers; (190.4420) Nonlinear optics, transverse effects in; (190.3270) Nonlinear optics, Kerr effect; (190.4223) Nonlinear wave mixing.
\end{abstract}

Multimode optical fibers (MMFs) had so far limited applications, mainly because of their inherent inability to maintain a good beam quality due to mode scrambling. In recent years however, nonlinear MMFs have emerged as an easily accessible playground to explore complex spatio-temporal optical pulse propagation phenomena [1]. In the anomalous dispersion regime, femtosecond multimode optical solitons have been observed in graded-index (GRIN) MMFs [2]. Their longitudinal periodic intensity oscillations have led to the controlled generation of ultra-wideband dispersive wave sideband series $[3,4]$. Whereas in the normal dispersion and with picosecond to nanosecond pump pulses, sideband series spanning from the visible till the mid-infrared (MIR) have been generated, resulting from the modulation instability of quasi-continuous waves, induced by the periodic beam self-imaging effect [5,6]. Fardetuned parametric frequency conversion was also achieved in a few mode GRIN fiber pumped at $1064 \mathrm{~nm}$ : multiple sidebands spanning in the visible down to $405 \mathrm{~nm}$ and in the near infrared up to $1953 \mathrm{~nm}$ were generated via a complex cascaded process involving inter-modal four-wave mixing [7].

Before the onset of spatio-temporal instabilities, as shown in figures 1-2, the Kerr effect alone has been shown to produce, for peak powers above a certain threshold, unexpected spatial beam self-cleaning towards a well-defined, and robust bell-shaped transverse profile after propagation over about $1 \mathrm{~m}$ of GRIN MMF [8]. The interplay between Kerr effect and Raman scattering in GRIN MMF has been shown to generate a spectrally flat, and spatially single-mode supercontinuum extending from the visible till the MIR [9]. As shown in figure 2, a temporally resolved analysis reveals significant (tenfold) temporal compression in the spectral sidebands [9]. Spatial Kerrinduced beam self-cleaning was observed with increased efficiency (i.e., with input peak powers below $1 \mathrm{~kW}$ ) in an active, ytterbium doped MMF with a step-index profile [10]. Finally, by optical poling a GRIN MMF, we generated a photo-induced charge distribution and a permanent modulated quadratic nonlinearity, leading to quasi-phasematched second-harmonic (SH) generation. We observed that the self-imaging of the pump wave led to the generation of a spectral sideband series around the SH wave. Moreover, the simultaneous spatial self-cleaning of pump and SH beams was observed [11].

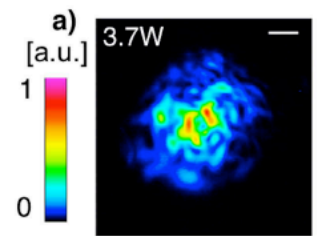

a')

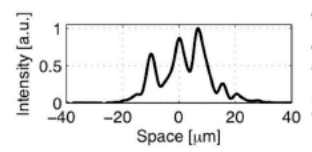

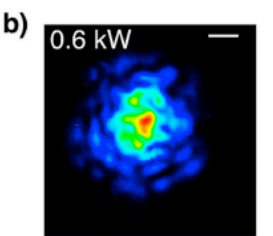

b')

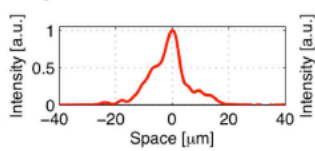

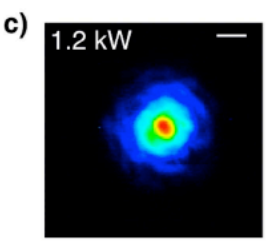

c')

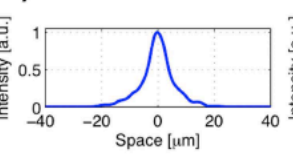

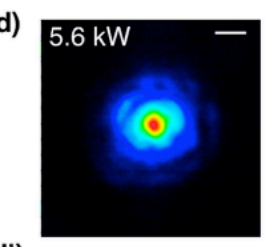

d')

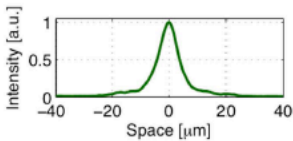

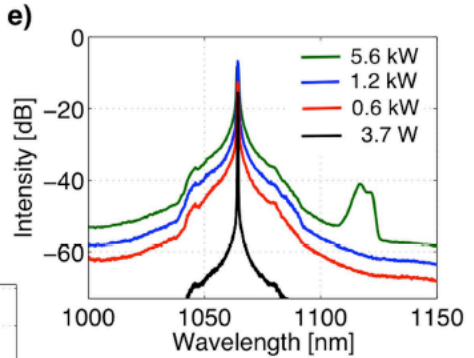

Fig. 1. Experimental demonstration of spatial Kerr beam self-cleaning. (a-d): Intensity profile of output transverse beam from $12 \mathrm{~m}$ of GRIN MMF for different peak pulse powers; (a'-d'): corresponding beam cross-sections; (e) output spectral intensity. 

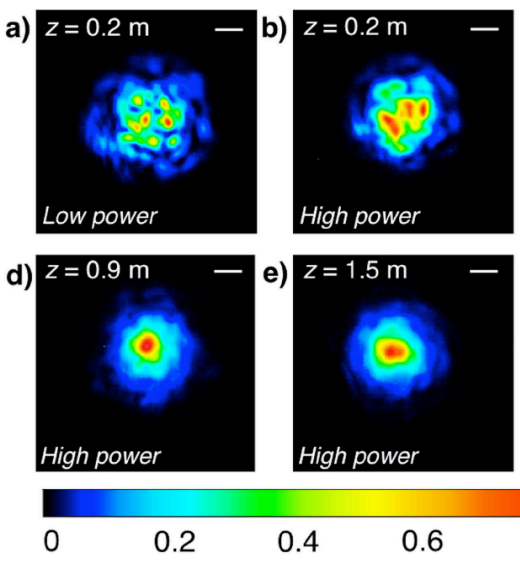
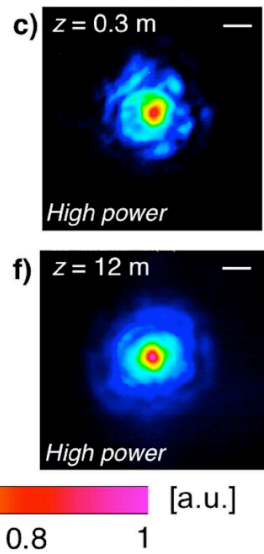

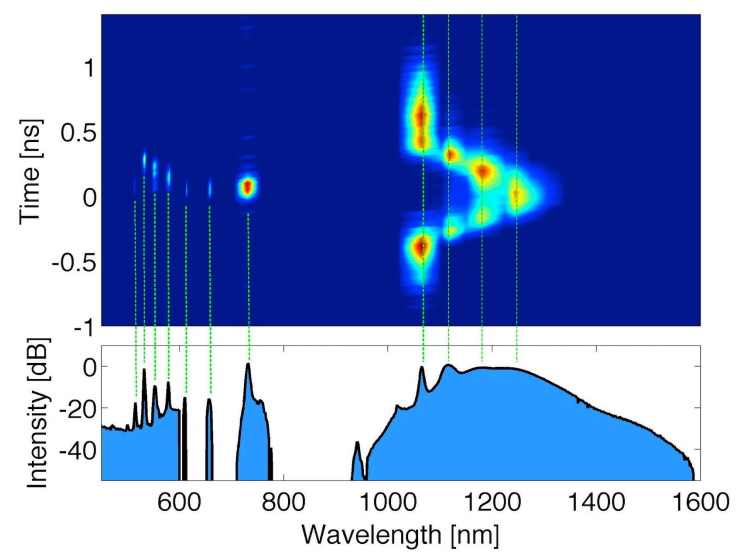

Fig. 2. (a-f) Intensity profile of output transverse beam from GRIN MMF for different fiber lengths at low power and high power, respectively; right panel: output spectral intensity profile (top) and spectrogram (bottom) showing sideband series and supercontinuum generation.

All of these observations may pave the way to the future development of a new class of photonic devices for a wealth of applications, based on combining an effective spatial single-mode environment with large fiber-core diameters.

\section{References}

[1] A. Picozzi, G. Millot, and S. Wabnitz, "Nonlinear virtues of multimode fibre," Nat. Photon. 9, 289-291 (2015) doi.org/10.1038/nphoton.2015.67.

[2] W. H. Renninger, and F. W. Wise, "Optical solitons in graded-index multimode fibres," Nat. Commun. 4 1719, (2013) doi.org/10.1038/ncomms2739.

[3] L. G. Wright, D. N. Christodoulides, and F. W. Wise, "Controllable spatiotemporal nonlinear effects in multimode fibres," Nat. Photon. 9, 306-310 (2015) doi.org/10.1038/nphoton.2015.61.

[4] L. G. Wright, S. Wabnitz, D. N. Christodoulides, and F. W. Wise, "Ultrabroadband Dispersive Radiation by Spatiotemporal Oscillation of Multimode Waves,” Phys. Rev. Lett. 115, 223902 (2015) doi.org/10.1103/PhysRevLett.115.223902.

[5] K. Krupa, A. Tonello, A. Barthélémy, V. Couderc, B. Mohamed Shalaby, A. Bendahmane, G. Millot, and S. Wabnitz, "Observation of geometric parametric instability induced by the periodic spatial self-imaging of multimode waves," Phys. Rev. Lett. 116, 183901 (2016) doi.org/10.1103/PhysRevLett.116.183901.

[6] L. G. Wright, Z. Liu, D. A. Nolan, M. Jun Li, D. N. Christodoulides and F. W. Wise, "Self-organized instability in graded index multimode fibre," Nat. Photon. 10, 771-776 (2016) doi.org:10.1038/nphoton.2016.227.

[7] R. Dupiol, A. Bendahmane, K. Krupa, A. Tonello, M. Fabert, B. Kibler, T. Sylvestre, A. Barthelemy, V. Couderc, S. Wabnitz, and G. Millot, "Far-detuned cascaded intermodal four-wave mixing in a multimode fiber," Optics Letters 42, 1293-1296 (2017) doi.org/10.1364/OL.42.001293

[8] K. Krupa, A. Tonello, B. M. Shalaby, M. Fabert, A. Barthélémy, G. Millot, S. Wabnitz, V. Couderc, "Spatial beam self-cleaning in multimode fiber," arXiv.org/abs/1603.02972 [physics.optics], and Nat. Photon. (2017) doi.org: 10.1038/nphoton.2017.32.

[9] K. Krupa, C. Louot, V. Couderc, M. Fabert, R. Guenard, B. M. Shalaby, A. Tonello, D. Pagnoux, P. Leproux, A. Bendahmane, R. Dupiol, G. Millot, and S. Wabnitz, "Spatiotemporal characterization of supercontinuum extending from the visible to the mid-infrared in multimode gradedindex optical fiber," Opt. Lett. 41, 5785-5788 (2016) doi.org/10.1364/OL.41.005785.

[10] R. Guenard, K. Krupa, R. Dupiol, M. Fabert, A. Bendahmane, V. Kermene, A. Desfarges-Berthelemot, J. L. Auguste, A.Tonello, A. Barthélémy, G.Millot, S. Wabnitz, and V. Couderc, "Kerr self-cleaning of pulsed beam in an ytterbium doped multimode fiber," Optics Express 25, 4783-4792 (2017) doi.org/10.1364/OE.25.004783

[11] D. Ceoldo, K. Krupa, A. Tonello, V. Couderc, D. Modotto U. Minoni, G. Millot, and S. Wabnitz, "Second harmonic generation in multimode graded-index fibers: spatial beam cleaning and multiple harmonic sideband generation," Optics Letters 42, 971-974 (2017) doi.org/10.1364/OL.42.000971 